\title{
Genetic Algorithm based Optimized Image Registration using Fast Walsh Hadamard Transform
}

\author{
Dr. Sasikala $\mathrm{D}^{1}$, Dr. Neelaveni $\mathrm{R}^{2}$ \\ ${ }^{1}$ Professor, CVR College of Engineering/CSE Department, Hyderabad, India \\ Email: anjansasikala@cvr.ac.in \\ ${ }^{2}$ Professor, PSG College of Technology/EEE Department, Coimbatore, India \\ Email: rnv.eee@psgtech.ac.in
}

\begin{abstract}
Numerous medical means are mandatory with accuracy and totality of data about a patient for a doctor for healthcare predictive analytics and so as to treat and take care of their patients' remedy. Medical image registration practices bestow more affluent diagnosis and medication information to the doctors in stipulating an all-embracing allusion resource for the beneficiaries entangled in image registration as an optimization crisis. In this research work, optimization of the Genetic Algorithm based image registration technique using Fast Walsh Hadamard Transform was implemented and their evaluation of results proved to be good.
\end{abstract}

Index Terms: Medical Image Registration, Fast Walsh Hadamard Transform, Ratio of Image Uniformity (RIU), Optimization, Genetic Algorithm.

\section{INTRODUCTION}

Image registration in medicine is the resolution of spatial transformation or representation that associates locations in single image specifically, the archetype picture, to their related loci in one or additional imageries called altered images from the identical or dissimilar imaging modalities [1].

The usages of medical image registration include monomodal image registration of the alike patient for observing and enumerating the sickness growth all across the time, assessment of intra-operative brain warping and intramural-operational image that steered persuasion of stereotactic devices and multimodal image registration.

Fast Walsh-Hadamard transform (FWHT) is a real progression to figure the Walsh-Hadamard transform (WHT). A straightforward effecting of the WHT of instruction $\mathrm{n}=2^{\mathrm{m}}$ would take a computational impediment of $\mathrm{O}\left(\mathrm{n}^{2}\right)$. The FWHT demands merely $\mathrm{n} \log$ $\mathrm{n}$ additions or subtractions. The FWHT is a decrease and overcome practice that is recursively splitting downhill a WHT of size $n$ obsessed by two compact WHTs of size $n / 2$. This procedure tag on the recursive portrayal of the $2^{\mathrm{m}} \times 2^{\mathrm{m}}$ Hadamard matrix :

$$
H_{m}=\frac{1}{\sqrt{2}}\left(\begin{array}{cc}
H_{m-1} & H_{m-1} \\
H_{m-1} & -H_{m-1}
\end{array}\right)
$$

The $1 / \sqrt{2}$ standardization parameters for each phase can

be clustered mutually or still ignored.

This sequence ordered matrix, also known as Walsh ordered, Fast Walsh-Hadamard transform, FWHT, is acquired by calculating the FWHT as above, and then reordering the productions.

A modest fast non-recursive operation of the WalshHadamard transform trails from disintegration of the Hadamard transform matrix as $\mathrm{H}_{\mathrm{m}}=\mathrm{A}^{\mathrm{m}}$, where $\mathrm{A}$ is $\mathrm{m}^{\text {th }}$ basis of $\mathrm{H}_{\mathrm{m}}[2]$.

Optimization in image registration by means of Genetic Algorithm (GA) is to establish the conversion factors namely translation, rotation and scaling that score the least denomination of a space computation that is one of the intents of image registration [3]. The accomplishment in creation of the best or utmost good function of a status quo encompasses realizing the best factors corresponding to an intent function that is moreover decreased or increased.

Fast Walsh Hadamard transform is more reliable in medical image registration consuming less time [4].

Performance Metrics: Ratio of Image Uniformity (RIU) is castoff so as to qualitatively evaluate the execution of the algorithms and associate with prevailing algorithms. To compute the similarity of images similarity measures are manipulated. The selection of similarity measure be influenced by the modality of images to be registered. Ratio of image uniformity are exercised for monomodal image registration.

This work deliberates the purpose of Genetic Algorithm for the choice of transformation parameters $(x, y, \theta, s)$ to be applied in image registration using FWHT algorithm. Analysis was carried out with the medical images and the best outcomes for registration of monomodal images are acquired.

\section{REVIEW OF LITERATURE}

Genetic algorithm is portrayed by [5] for the deconvolution drawbacks of image restoration and it is demonstrated as an optimization challenge, whose price function is reduced established on system of natural selection and natural genetics.

Venkateshwara Rao $\mathrm{Ch}$ et al instigated and estimated a group of programmed registration processes to rectify the geometric faults of the input image with respect to the altered image, by amplifying the precision level of the registration and minimizing the Root Mean Square (RMS) error to less than a pixel. Numerous processes such as Wavelet Transformation method, Fast Fourier Transformation method, Morphological Pyramid approach and Genetic Algorithms were established and evaluated. 
These processes ponder the alteration model to sub-pixel accuracy [6].

Medical images of CT, PET or MRI images were wellthought-out for investigation. But the algorithm conferred involves both the images to be of the identical scale. ChungHsien Huang et al anticipated a registration technique established on adaptive genetic algorithm to accomplish the registration of $\mathrm{CT}$ and facial surface data. The writers applied chain code scheme to lessen a big quantity of surface data and decreased the decision-making time proficiently. With the idea of the genetic algorithm in continuous space (GACS), enhancement in evolutional processes of chromosomes crossover and mutation was accomplished to hurry up the convergent process of GA. The registration technique anticipated by the writers can be castoff for non-fiducial, stereotactic brain surgeries and aid surgeons to analyze and heal brain illness precisely and easily [7].

Marcus Johansson rendered outcomes of both the genetic algorithm and simulated annealing optimization techniques as viable substitutes for execution of image registration. Assessments carried out by the writer specified zero-error registrations with four renovations [8].

Flavio Luiz Seixas et al focused on the image registration complexity by harnessing the genetic algorithm background. The instigators concentrated on the point matching setback with a technique built on nearest-neighbor [9].

In this script, the generative model for affine transformations on image points have been pondered. How the amalgamation of suitable priors of the transformation and noise into the generative model have been defined steers to the improved assessments. The usage of these approximators are exhibited on the complications of object recognition, image registration and comparison. It is perceived that the Bayesian technique outshines all additional approaches and this fabrication of the probabilistic invariant is desirable upon others [10].

Explorers propounded an innovative technique for the intermodal registration of images by means of a measure Mutual Information and they powerfully emphasize that their solutions are outstanding than the rest of the works as their outcomes such as robustness, accuracy is proved to be utmost high and mighty [11].

In this research, a newfangled automated system to register retinal images was described and tested in a medical atmosphere in view of the consistency, suitability and efficiency of diverse image transformation models and function optimization methods, succeeding an opening preprocessing phase. Three dissimilar transformation models-affine, bilinear and projective-in addition to three optimization schemes-downhill simplex method, simulated annealing and genetic algorithms-are explored and associated in terms of accuracy and efficiency [12].

This artefact conferred the traditional approaches in image registration as of the characteristics of cross-modal adaptation and geometric renovation of an image, measurement of similarity, repetition and optimization, furthermore to additional sections. In the arena of medical image investigation, image registration endures as one of the maximum explored topics. Yet, numerous complications persist to be resolute in this zone. A unique complexity is that medical images are typically grayscale images that orders advanced necessities in parameter optimization and modifications. Further hindrance is that the way in which to stimulate the accuracy and speed of registration. Likewise, the robust is one of the drawbacks that obstructs the progress of registration. Promoting research in this coverage area must concentrate on success by means of this inadequate data in handling high-accuracy registration, whereas decreasing the registration and computation time to intensify the time sensitivity of registrations [13].

The Optimized Automatic Image Registration scheme in MIPAV dominates by the key tactic for ascertaining a global optimal renovation in estimating a minimal price function, and maximal resolutions between the reference and target images at numerous dissimilar resolutions opening with the least resolution. Each step of escalating resolution operates with the formerly verified optimal transformation as an initial point and further hones its estimates. This technique frequently works fine with the images of the same modality [14].

In this research three pioneering registration approaches were conceived with the interpretations of the mutual information and optimization technique: (1) mutual information pooled with the downhill simplex method of optimization. (2) the derived mutual information fused with Quasi-Newton method. (3) mutual information blended with hybrid genetic algorithm (significant- universe brute-force search) to evade local ceiling all through the optimization. These instinctive registration classifications went through assessments by a series of images, magnitudes and voxel resolutions. Experimentations reveal that registration logic linked with mutual information and hybrid genetic algorithm can stipulate strong and precise orientations to attain a complex triggering diagram and representation for practical MRI laboratory assay.

To probe the most efficient techniques and to inspect the level of advancement attainable by augmenting them with Wavelet transform. The SIFT feature-based scheme services the Eigen appraisal for extricating thousands of significant facts created on scale invariant facts and these fact points once extended is heightened by the wavelet transform relinquishes the paramount outcomes [15].

In this research article, a mono modal image registration algorithm is completed for the alignment of T1-weighted MR images of human brain using modified Particle Swarm Optimization (PSO) technique for attainment of the best spatial coordinates of the stirring image. The investigational outcomes evidently illustrate that the anticipated algorithm assurances improved findings than the established PSO algorithm [16].

Discrete optimization propounds appealing proficiencies for non-rigid medical image registration. Reconstructed a creative non-rigid registration technique established on the preceding effort on stereo vision with huge displacement spaces. The foremost notion is to reduce the complication in working out to the persistent time complexity is to lessen the exploration space hierarchically from coarse to fine levels. The huge search space supports in keeping away from local minima in the optimization scheme. Currently this technique 
is practical to a major extent in resolving demanding registration tasks pervasively and ubiquitously. This method associates very positively besides state-of-the-art registration methodologies [17].

Georeferencing images is a painstaking method thus systems for systematizing this method have been underneath research for certain period. Amongst the utmost favorable programmed registration algorithms are those built on Fast Fourier Transform (FFT). The shift concerning the two specified images can be calculated by estimating the fraction $\mathrm{F} 1 * \operatorname{conj}(\mathrm{F} 2) /|\mathrm{F} 1 * \mathrm{~F} 2|$, and then employing the inverse Fourier transform. The outcome is an impulse-like function that is almost zero ubiquitously excluding at the shift that is essential to optimally register the images. Renovating from rectangular coordinates to log-polar coordinates, shifts signifying rotation and scaling too can be too firm to accomplish the georectification process. This FFT-based algorithm has persisted magnificently realized in IDL Interactive Data Language (ID) and appended as two user roles to an image processing software package ENvironment for Visualizing Images (ENVI) overlapping facts. ENVI dials all pre-processing and post-processing task for instance- input, output, display, filter, analysis, and file management. For assessment of this enactment, numerous dozen assessments were steered in cooperation of replicated and "physical world" imageries. The outcomes of these assessments exhibit advantages and restrictions of this algorithm. In precise, these assessments display that the precision of the subsequent registration is fairly virtuous measured up to the present physical approaches.

Wavelet Modulus Maxima process approve to grasp the images are of matching resolution. In this technique threshold factors requires to be collaboratively stipulated. Owing to its pyramidal style consents for quicker execution and sophisticated registering accuracy. It is further suitable to register images engaged commencing from the identical sensor. It operated sound for imageries acquired at dissimilar times that are distinctive to remote sensing purposes. As this utilizes the control points scheme it can correct the local faults that outdoes manual registration of images. FFT method delivers precision adequately worthy. The procedure operates for images in which the scale transformation is not as much of than 1.8 [18].

Owed to the universal transform this method cannot influence local geometric misrepresentations. The Modulus Maxima Process image registration algorithm with an intensity-based differential matching method is consistent and well-organized accomplished for computing the faults, to sub pixel precision, the movement amongst images exposed to affine transformation that embraces concurrent translation, rotation, scaling, and shearing. GAs can proficiently explore the solution space and stretches the solution in accomplishing the sub pixel precision without recognizing the control points. Over global transformation an archetypal can be instituted for translation and rotation faults. The anticipated algorithm assumes that together the images are of identical scale. Computational proficiency can be enhanced by espousing the pyramidal method. Liable to the type of discrepancies in the medical images of Computerized Tomography, PET or MRI images a few of these methods can be approved for assembling the numerous interpretations. It is improbable that a single registration system will operate reasonably. To portray these algorithms the shared data sets from IRS PAN are castoff and there is no scale dissimilarity [19].

\section{GENETIC AlgorithM}

Amongst several approaches to image registration challenge from the metaheuristics line of reasoning, genetic algorithms (GAs) [5, 6, 7, 8, 9] is very well-organized and valuable. GA's are computational representations of natural evolution in which robust persons are further liable to be the champions in a cutthroat atmosphere. It is a stochastic technique be responsible for a decent deed in gaining knowledge of factors.

\section{A. Genetic Algorithm Based Optimization for Monomodal Image Registration Process}

Algorithm: $\mathrm{Be}$ concerned about $\mathrm{X}$ and $\mathrm{Y}$ as the two images with $2 \mathrm{D}$ points. $\mathrm{X}$ is the archetype image and $\mathrm{Y}$ is the altered image acquired from $X$ after affine transformation. The optimized image registration classification comprises of an optimizer that decreases RIU computation.

Step 1: Key in image X

Step 2: Achieve affine transformation built on the chromosome value and get the altered image.

Step 3: Accomplish optimized registration using FWHT algorithm and figure out RIU. RIU decreases the deviation between archetype and altered image.

Step 4: Iterate Steps 2 and 3 for all adherents of the residents.

Step 5: Test if the quantity of productions is 100 . If as a result, end otherwise employ genetic factors to materialize the innovative residents and Iterate Step 2 to Step 5.

Figure 1. signifies fitness estimation order, where $\mathrm{x}_{\mathrm{i}}, \mathrm{i}=$ $1,2, \ldots \mathrm{N}$, is the archetype fact realized by the spatial transformation characterized by affine matrix factors. The feasible resolutions are signified as a chromosome vector characterized by four genes, reproducing translation $\left(t_{x}, t_{y}\right)$, rotation $(\theta)$ and scaling (s) impacts, for example exhibited by Figure 1 .

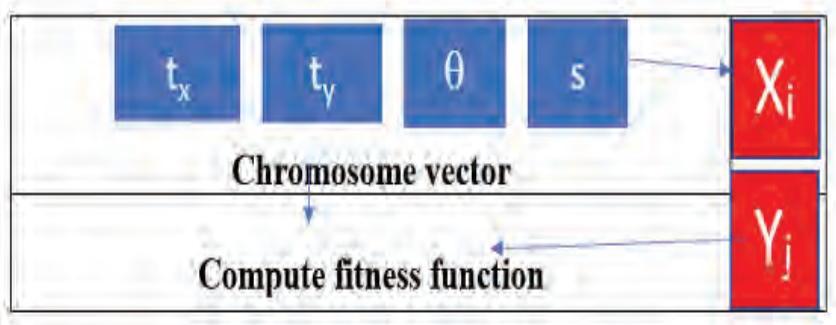

Figure 1. Fitness computing scheme - Fitness evaluation sequence

These spatial transformations are parameterized in affine matrix. The GA's aim is to discover the elite spatial transformation (chromosome) in the individual residents. 


\section{i. GA Operators}

Three genetic operators (selection, crossover and mutation) are achieved on the entire inhabitants to influence the chromosomes to the optimal resolution.

\section{Selection}

Using a random selection system with roulette wheel selection method, the chromosomes (estimates) with lower RIU (higher fitness) are carefully chosen and reproduced precisely in the novel cohort deprived of every deviation. The chromosomes (estimates) with higher RIU (lower fitness) are swapped with offspring (new-fangled estimates) shaped by the parents that are chosen randomly, with crossover and mutation.

\section{Crossover}

A crossover operator is castoff for creation of descendants. The crossover process is achieved for every row or every column as specified below:

Step 1: Randomly opt for an integral figure amongst of 0 and 1 to ascertain the row or the column (If the reimbursed value is 0 at that moment the crossover process is accomplished for all row, else the crossover process is executed for every single column).

Step 2: Randomly pick out an actual value as of the range $[0.0,1.0]$

Step 3: If the reverted value is equal to or less than the likelihood of crossover ( 0.5 in the simulations) then duplicate the row/column from the parent 1 to the descendants, otherwise duplicate the row/column from the parent 2 to the descendants.

Step 4: Step 2 and Step 3 are harnessed to each and every row or column.

\section{Mutation}

Only mutation approach is manipulated for fabrication of descendants in concert with the crossover. The procedure is primed as listed beneath:

Step 1: Arbitrarily choose a tangible value from the extent $[0.0,1.0]$.

Step 2: If the recompensed value is equal to or less than the conferred likelihood of mutation $(0.02$ in the simulations), then the pixel value is mutated from 0 to 1 or from 1 to 0 , if not the pixel value is untouched.

Step 3: Step 1 and Step 2 are put into operation to every pixel.

The likelihood of mutation is a significant factor that extensively alters the efficiency of the algorithm. It is found to be inversely relational to the quantity of pixels of the image.

A Fast Walsh Hadamard Transform (FWHT) was anticipated in this research for medical image registration $[20,21]$. This transform lessens the time usage in image registration. So, it shows to be an improved method for medical image registration than any further traditional Walsh Transform. The factors attained by means of this transform are then stabilized to acquire the exceptional number. This number signifies the local edifice of an image. Besides, this it also directs the highlights of an image for image registration. Though the investigational consequences exposed the details that the projected algorithm using Fast Walsh Hadamard Transform achieved good image registration, this current work focuses on additional enhancement in the outcomes by using Genetic Algorithm Based Image Registration.

Here while performing optimization of image registration using FWHT considering coefficients GA Operators - $t_{x}, t_{y}, \theta$ and $\mathrm{s}$, after optimization it exhibited that RIU has been reduced by minimizing the fitness function.

The future work is to be pondered on surplus improvement in the outcomes with optimization of image registration using FWHT considering the same GA Operator quantities - $t_{x}, t_{y}, \theta$ and $s$, and to explore that after optimization it reveals that Correlation Coefficient has been increased by maximizing the fitness function.

\section{Simulation RESULTS}

For investigation, MRI-T2 Sagittal brain image is pondered. The earliest size of these images is specified in pixels. In order to eliminate the environment of the image and the head sketch out, the archetype images and are trimmed based on the Region Of Interest (ROI), conceiving sub-images of diverse measured pixels.

TABLE I.

PERFORMANCE COMPARISON OF RIU FOR IMAGE REGISTRATION USING FWHT BASE 8 FOR MONOMODAL IMAGES

\begin{tabular}{|c|c|c|c|c|c|}
\hline \multicolumn{3}{|c|}{ Before Registration } & \multicolumn{2}{|c|}{ FWHT Base 8 } \\
\cline { 3 - 6 } $\mathrm{x}$ & $\mathrm{y}$ & $\begin{array}{c}\theta \\
\text { degree }\end{array}$ & $\mathrm{s}$ & $\begin{array}{c}\text { Before } \\
\text { Optimization }\end{array}$ & $\begin{array}{c}\text { After } \\
\text { Optimization }\end{array}$ \\
\hline 0 & 0 & 270 & 1 & 0.6013 & 0.5861 \\
\hline
\end{tabular}

GA based optimization is performed using FWHT Base 8 algorithm. An early population size of 10 chromosomes is measured with $\mathrm{x}, \mathrm{y}$ fluctuating between -25 to $+25, \theta$ varying in the interval -180 to +180 and scaling interval is fixed as 0.5 to 1.5 . RIU is well-thought-out as the fitness function, subsequently it computes the degree of similarity relating two images.

Table I lists the optimized value of transformation parameters for Image Registration using FWHT Base 8 algorithm with RIU as fitness function that is decreased, and it can be noted from Table II.

TABLE II.

OPTIMIZED TRANSFORMATION PARAMETERS FOR IMAGE REGISTRATION USING FWHT BASE 8 TECHNIQUE

\begin{tabular}{|l|l|c|l|}
\hline \multicolumn{4}{|c|}{ Before Registration } \\
\hline $\mathrm{x}$ & $\mathrm{y}$ & $\begin{array}{c}\theta \\
\text { degree }\end{array}$ & $\mathrm{s}$ \\
\hline 21 & 16 & -5 & 1 \\
\hline
\end{tabular}




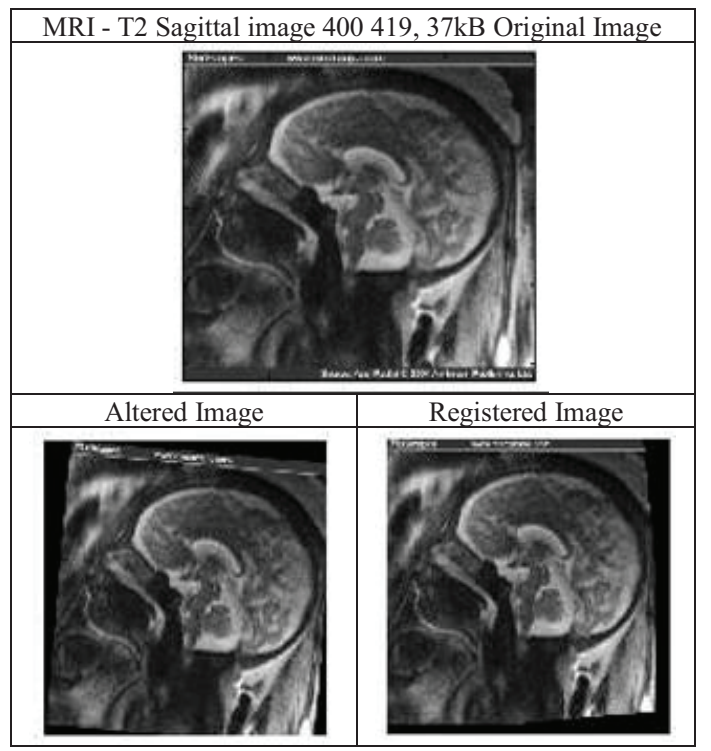

Figure 2. MRI - T2 sagittal $400419,37 \mathrm{kB}$ jpg image before GA based optimization for image registration using FWHT Base 8 technique with

$$
(\mathrm{x}=0, \mathrm{y}=0, \theta=270, \mathrm{~s}=1)
$$

Figure 2. depicts the image registration of MRI - T2 Sagittal image before optimization with transformation parameters as $(x=0, y=0, \theta=270, s=1)$.

Figure 3 . shows the registration of the same image after optimization with transformation obtained as $(\mathrm{x}=21, \mathrm{y}=$ $16, \theta=-5, \mathrm{~s}=1)$. It is observed that better registration result is obtained after optimization.

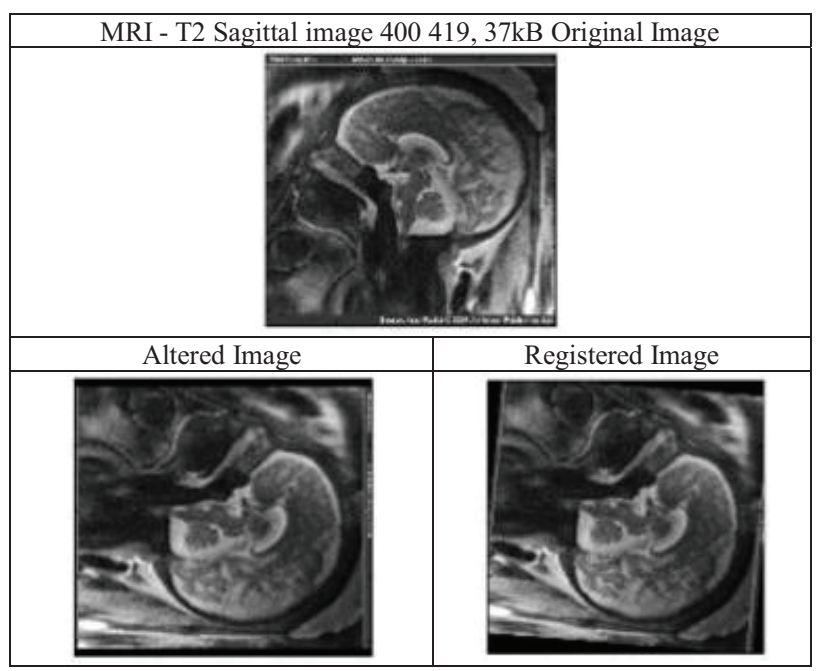

Figure 3. MRI - T2 sagittal $400419,37 \mathrm{kB}$ jpg image after GA based optimization for image registration using FWHT Base 8 technique with $(x=21, y=16, \theta=-5, s=1)$

\section{SUMMARY}

This simulation proves that Optimized image registration using FWHT is better one as RIU values are reduced.

Based on the observations it is noted that the usage of these transformations to the algorithm yielded MI as 3.6536 and $\mathrm{CC}$ as 0.7424 . This revealed an augmentation of $80.05 \%$ and $36.23 \%$ respectively.

\section{CONCLUSIONS \& FUTURE WORK}

Fast transforms are utilized to create the algorithms to be working out truthfully and vigorously and correspondingly to recuperate the data alignment. Likewise, devotion of Genetic Algorithm (GA) for the augmented preference of translation, rotation and scaling quantities enriching the similarity measures is achieved. The elevated categorization of methods as soon as they are suitable, are habitually further disciplined in their practice of approximation resolution than the image registration and its optimization approaches. Hitherto, when few of the images are unavailable, problematic or posh to attain, or is expected to be untrustworthy, higher-order procedures that naturally form a single registration objective built on the optimization, can change to complications from being misregistered. The abilities of image registration and their optimization methodologies are that even though they are optimizationally-correlated, they are not lay open to the optimization and, they anticipate on plentiful tracks. This may not be further effectual, but it can be abundantly reliable in such state of affairs. Unique and further traits of optimization approach is that as they enhance in frequent tracks, almost limited of this can sound in the mounting channels, they can be treasured in environments everywhere there are an immeasurable resident that decrease RIU. However, this is a sensible preliminary learning, and have confidence in the aforementioned optimization of RIU measure that such developments would be additional motivation in the realism of optimization design practices in the framework of putting an end to the multi-disciplinary design optimization complications too.

The following are a small number of recommendations put forward for future work.

- The alteration systems castoff in the research can be broadened to small and large size images too.

- As per a future work, the systems can be employed for dissimilar image formats and performance metrics can be analyzed.

- Image registration methods conferred in this research study can be harnessed for additional modalities like PET, SPECT, etc.

- The systems may be assessed by utilizing other renovations that embrace shearing and reflection too.

- The task described in the research can be stretched out for fusion of brain images as well.

- The methods may be outspread for registration of other parts of bodies that take in heart, kidney, lungs etc.

- Genetic Algorithm can correspondingly be castoff to boost Mutual Information (MI) for monomodal and multimodal images.

- Optimized renovations can likewise be attained for GA Based Image Registration Techniques Using Modified Adaptive Polar Transform (MAPT) and Wang Landau Adaptive Monte Carlo (WLAMC) methods. 
In the practices bestowed directly above, the metrics are estimated are centered on quantities of translational factors $\mathrm{x}, \mathrm{y}$; rotational factor, and scaling factor s selected on trial and error. The image registration algorithm is optimized to advance the registration progression by instigating Genetic Algorithm process of Image Registration using Fast Walsh Hadamard Transform (FWHT) [9] to cater GA based Optimized image registration using FWHT system. Ratio of Image Uniformity (RIU) is resolute to authorize the enhancement in registration.

\section{REFERENCES}

[1] Barbara Zitová and Jan Flusser, "Image registration methods: a survey", Image and Vision Computing, Elsevier, ScienceDirect, vol. 21, no. 11, pp. 977-1000, October 2003.

[2] B.J. Fino and V.R. Algazi, "Unified Matrix Treatment of the Fast Walsh-Hadamard Transform", IEEE Transactions on Computers, vol. 25, no. 11, pp. 1142-1146, November 1976.

[3] J.-M. Rouet, J.-J. Jacq, and C. Roux, "Genetic algorithms for a robust 3-D MR-CT registration", IEEE Transactions on Information Technology in Biomedicine, vol. 4, no. 2, pp. 126-136, June 2000.

[4] D. Sasikala and R. Neelaveni, "Registration of Brain Images using Fast Walsh Hadamard Transform", International Journal of Computer Science and Information Security (IJCSIS), vol. 8, no. 2, pp. 96-105, May 2010.

[5] Yen-Wei Chen, Zensho Nakao, and Xue Fang "Parallelization of a Genetic Algorithm for Image Restoration and its Performance Analysis", ICEC'96, 20th-22nd May, Nagoya, Japan, 1996.

[6] Venkateswara Rao Ch, Rao, K.M.M., Manjunath, A.S. and Srinivas, R.V.N. "Optimization of Automatic Image Registration Algorithms and Characterization", ISPRS'04, 12th--23rd July, Istanbul, Turkey, Vol. 35, 2004.

[7] Chung-Hsien Huang, Jiann-Der Lee and Jau-Hua Huang "Registration of CT Image and Facial Surface Data using Adaptive Genetic Algorithm", Biomedical Engineering: Applications, Basis and Communications, Published by World Scientific, Vol. 17, No. 4, pp. 201-206, 2005.

[8] Marcus Johansson, "Image Registration with Simulated Annealing and Genetic Algorithms", MS Thesis, Stockholm, Sweden, 2006.

[9] Flavio Luiz Seixas, Luiz Satoru Ochi, Aura Conci and Debora C. Muchaluat Saade "Image Registration using Genetic Algorithm", Proceedings of the 10th Annual Conference on Genetic and Evolutionary Computation Conference, GECCO'08, 12-16th July, Atlanta, Georgia, USA, ACM, pp. 1145-1146, 2008.

[10] Venu Madhav Govindu, and Michael Werman, "On using priors in affine matching", Image and Vision Computing, Elsevier, ScienceDirect, vol. 22, no. 2004, pp. 1157-1164, 2004.

[11] P. Thevenaz, and M. Unser, "Optimization of mutual information for multiresolution image registration", IEEE Transactions on Image Processing, vol. 9, no. 12, pp. 20832099, Dec, 2000.

[12] G.K. Matsopoulos, N.A. Mouravliansky, K.K. Delibasis, and K.S. Nikita, "Automatic retinal image registration scheme using global optimization techniques", IEEE Transactions on Information Technology in Biomedicine, vol. 3, no. 1, pp. 4760, March 1999.
[13] Guoli Song, Jianda Han, Yiwen Zhao, Zheng Wang and Huibin Du, "A Review on Medical Image Registration as an Optimization Problem", Current Medical Imaging Reviews, Bentham Open Access, vol. 13, no.3, pp.274-283, Aug 2017.

[14] Hongliang Yu, "Automatic Rigid and Deformable Medical Image Registration", A Dissertation of the Worcester Polytechnic Institute, pp. 1-116, May 2005.

[15] Arun P.V, and S.K. Katiyar, "An investigation towards wavelet-based optimization of automatic image registration techniques", https://arxiv.org/pdf/1303.6927.

[16] PN Maddaiah, PN Pournami, and VK Govindan, "Optimization of Image Registration for Medical Image Analysis", International Journal of Computer Science and Information Technologies (IJCSIT), vol. 5, no. 3, pp. 33943398, 2014.

[17] Mattias Paul Heinrich, Mark Jenkinson, Michael Brady, and Julia A Schnabel, "Non-rigid image registration through efficient discrete optimization", Proceedings of $15^{\text {th }}$ Annual Conference on Medical Image Understanding and Analysis MIUA 2011, vol.2011, London, UK, July 14-15, 2011.

[18] H Xie, N Hicks, GR Keller, H Huang, and V Kreinovich, “An IDL/ENVI implementation of the FFT-based algorithm for automatic image registration", Journal of Computers \& Geosciences Elsevier, ScienceDirect, vol. 29, no. 8, pp. 10451055 , Oct 2003

[19] Ch. Venkateswara Rao, Dr. K.M.M. Rao, A.S. Manjunath, and R.V.N. Srinivas, "Optimization of Automatic Image Registration Algorithms and Characterization", The Proceedings of the 31th International Symposium of Remote Sensing of Environment Joint Conference, 5th International Symposium Remote Sensing of Urban Areas (URS 2005), Tempe, AZ, USA, March 14-16 2005.

[20] D.Sasikala and R.Neelaveni, "An Analysis of Registration of Brain Images using Fast Walsh Hadamard Transform", International Journal of Computer Applications, vol 13. no. 1, pp. 23-29, January 2011.

[21] D.Sasikala and R.Neelaveni, "Performance Analysis of Brain Image Registration Technique using Fast Walsh Hadamard Transform and Modified Adaptive Polar Transform", Journal of Scientific and Industrial Research, vol 70., pp 123-128, February 2011. 